\title{
Targeted stem Cells expressing TRAIL as a therapy for lung CAncer
}

\section{TACTICAL: a phase $1 /$ II trial}

\section{Introduction:}

Current treatments for advanced NSCLC are associated with significant toxicities and only improve life expectancy by a few months. With over $70 \%$ of patients presenting with advanced disease there is a pressing need for novel therapies.

TNF-related apoptosis inducing ligand (TRAIL) is an anti-cancer therapy which causes apoptosis in tumour cells leaving healthy cells unaffected. We previously transduced mesenchymal stromal cells (MSCs) with a lentiviral vector to express TRAIL (MSCTRAIL). These cells home to tumours and induce apoptosis selectively in cancer cells resulting in a reduction in tumour growth in multiple in vivo models.

We have produced a viable working bank of MSCTRAIL and are now undertaking a first-in-man clinical trial to assess the therapeutic efficacy of this genetically modified cell therapy in the treatment of metastatic lung adenocarcinoma.

A key barrier to translation for cellular therapy is the understanding of the cell journey after delivery. ${ }^{111}$ Indium-oxide is established for lymphocyte tracking but it has low sensitivity and is toxic to cells. ${ }^{89}$ Zirconium-oxine is a novel PET tracer which has better sensitivity and lower toxicity. We have shown MSCTRAIL can be radiolabelled with ${ }^{89} \mathrm{Zr}$ without effecting viability or therapeutic efficacy.

\section{Trial design:}

Phase I is a dose de-escalation study, phase II is multi-centre, single blinded, randomised, placebo controlled trial (figure 1a and 1b).

A first-in-man expansion cohort of 5 patients will received ${ }^{89}$ ZrMSCTRAIL in cycles 1 and 3 . They will undergo serial PET scanning allowing mapping of cell location and duration.

\section{Outcomes:}

Phase I primary outcome is safety and tolerability of MSCTRAIL.

Phase II primary outcome is tumour response rate by RECIST ( $v 1.1$ ) criteria 12 weeks

Secondary outcomes include, best overall response, duration of response, progression free survival and overall survival.

Future Work:

If MSCTRAIL is safe and effective we plan to expand into larger phase III trials. 


\section{TACTICAL \\ Bayesian de-escalation mCRM model}

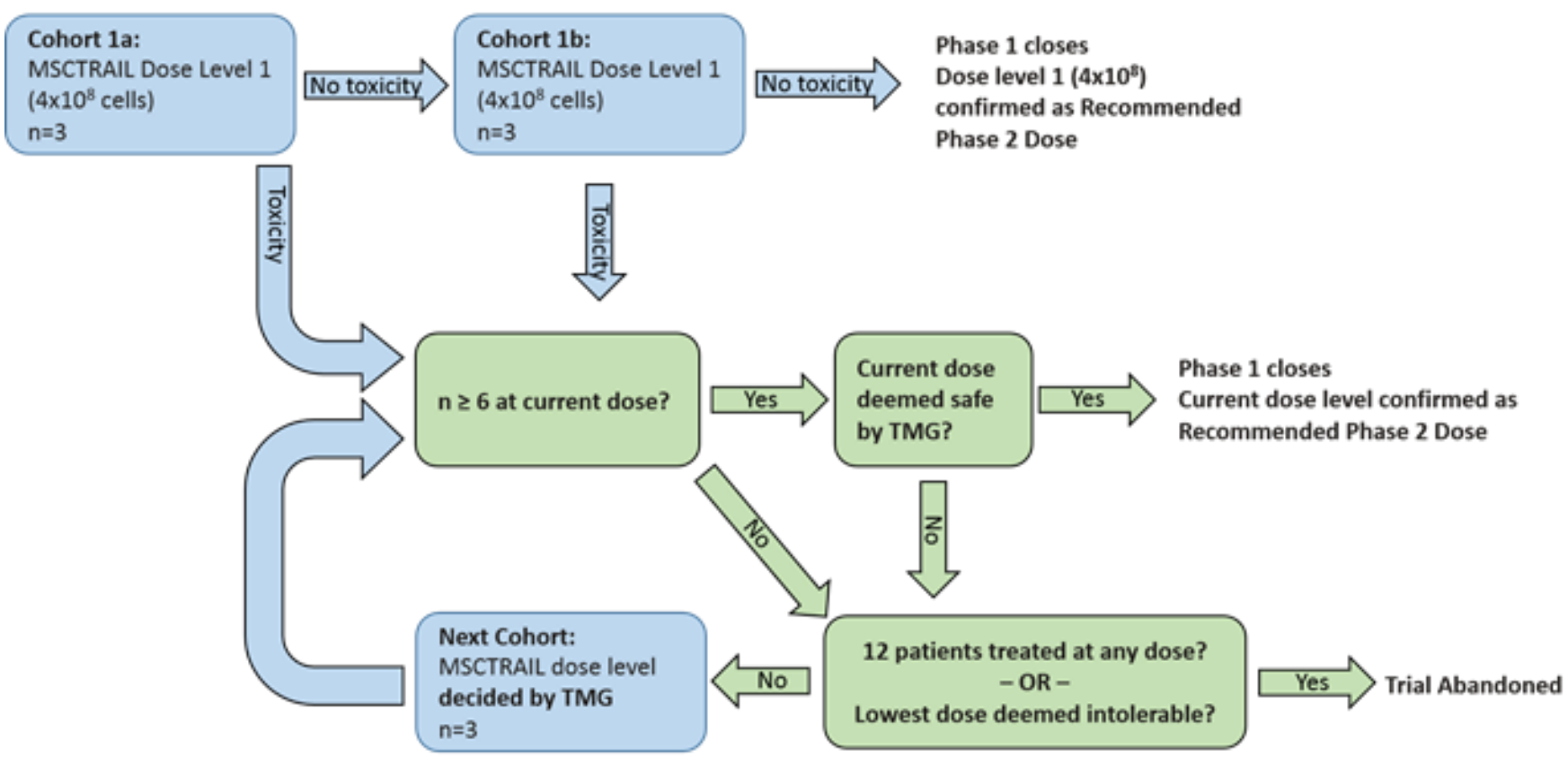

Figure 1b: Phase II Schema

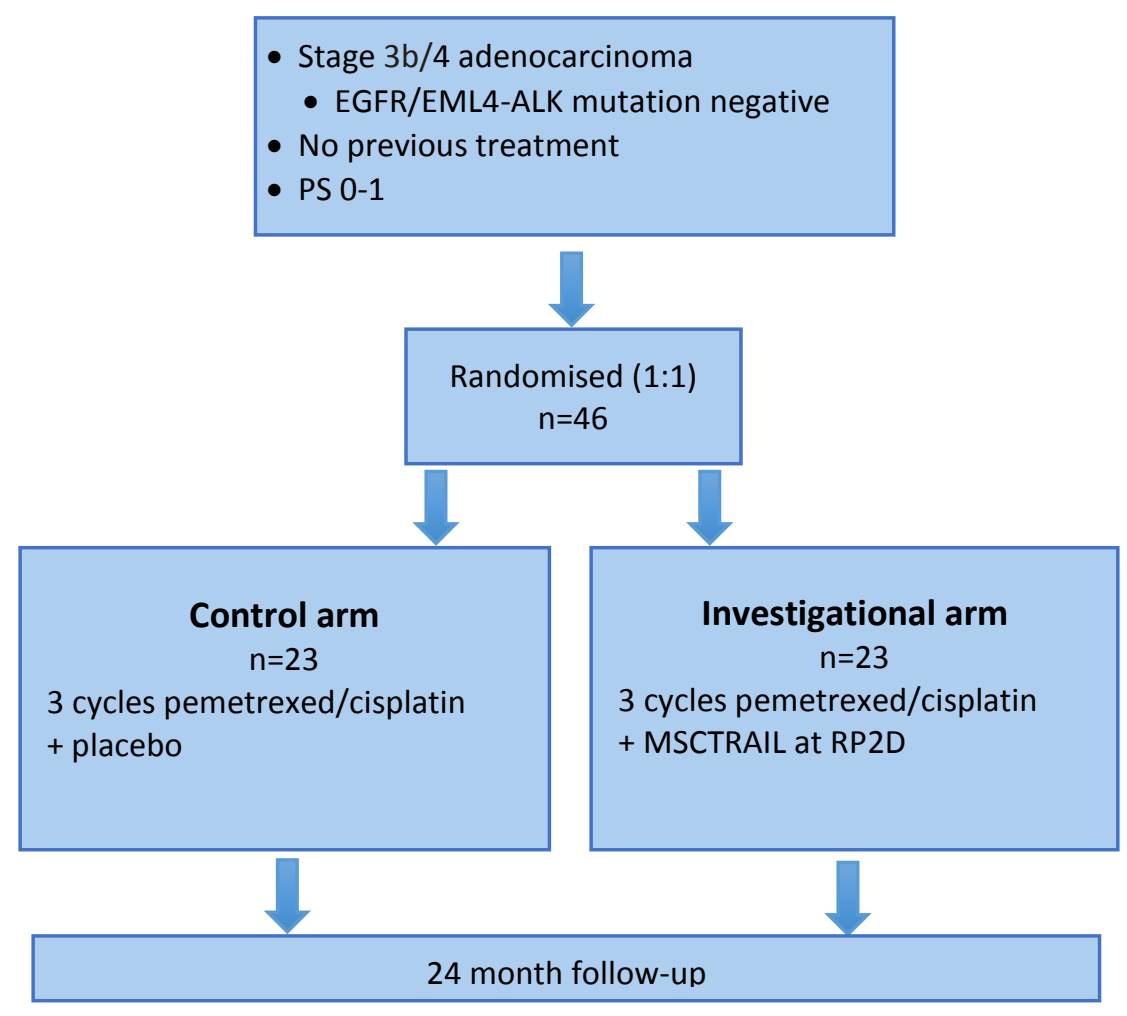


